(cc) $\mathrm{BY}-\mathrm{NC}-\mathrm{SA}$

\title{
DIALOGANDO SOBRE INCONTINÊNCIA URINÁRIA FEMININA, QUALIDADE DE VIDA E POÚTICAS PÚBLICAS DE SAÚDE PARA A MULHER BRASILEIRA
}

\author{
DIALOGUING ABOUT FEMININE URINARY INCONTINENCE, QUALITY OF LIFE AND \\ PUBLIC HEALTH POLICIES FOR BRAZILIAN WOMEN
}

José Tadeu Carvalho Martins ${ }^{1}$

Valmin Ramos da Silva ${ }^{2}$

\begin{abstract}
RESUMO
Este artigo foi extraído da dissertação de mestrado "Proposta de diretriz clínica de atenção à mulher com incontinência urinária na política pública de saúde", defendida em 2015, que abordou em seu estudo a prevalência da incontinência urinária em mulheres. A extração aqui contida versa a respeito da patologia de incontinência urinária que incide na população feminina, discutindo os efeitos deste problema de saúde sobre a qualidade de vida das mulheres acometidas, contrapondo esta questão de saúde pública com as políticas públicas de saúde existentes. Trata-se de um diálogo conceitual que tem por objetivo fomentar a discussão acerca das políticas públicas para mulheres considerando seus ciclos de vida, partindo de um conceito de atenção integral à saúde da mulher. A revisão aqui contida foi baseada em documentos governamentais, livros e artigos que abordam o assunto de incontinência urinária, políticas públicas, políticas de saúde, saúde da mulher, e acesso à base de dados Scielo,
\end{abstract}

\footnotetext{
${ }^{1}$ Médico urologista e cirurgião geral, Membro Titular da Sociedade Brasileira de Urologia, Mestre em Políticas Públicas e Desenvolvimento Local; Doutorando em Uroginecologia pela Escola Paulista de Medicina da Universidade Federal de São Paulo, Professor de Técnica Operatória na Universidade de Vila Velha. Preceptor de Uroginecologia na Universidade Federal do Espirito Santo; Médico responsável do Departamento de Urodinâmica do Hospital Metropolitano da Grande Vitória, Espirito Santo. Escola Superior de Ciências da Santa Casa de Misericórdia de Vitória - EMESCAM, Espírito Santo, Brasil. Telefone: (27) 3324 5013. E-mail tadeucarvalho.jtcm@yahoo.com.

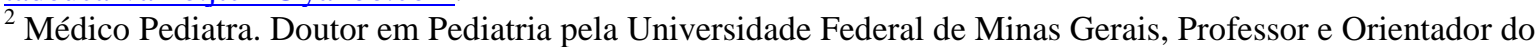
mestrado em Polícias Públicas e Desenvolvimento Local e professor da graduação do curso de Medicina da EMESCAM. Escola Superior de Ciências da Santa Casa de Misericórdia de Vitória - EMESCAM, Espírito Santo, Brasil. E-mail: valmin.silva@gmail.com
} 
Lilacs, PubMed. Conclui-se que a ineficiência das políticas públicas de enfoque à saúde da mulher aliado à dificuldade do sistema de saúde brasileiro, corroboram a um diagnóstico tardio e difícil acesso ao tratamento da patologia; elementos que contribuem para a deficiência na qualidade de vida das mulheres que sofrem de incontinência urinária.

PALAVRAS-CHAVE: Incontinência Urinária Feminina. Política Pública de Saúde. Qualidade de vida.

\section{ABSTRACT}

This article was extracted from the master's thesis "Proposal of a clinical guideline of attention to women with urinary incontinence in public health policy", defended in 2015, which addressed in their study the prevalence of urinary incontinence in women. The extract is related to the pathology of urinary incontinence that affects the female population, discussing the effects of this health problem on the quality of life of the women affected, opposing this public health issue with existing public health policies. It is a conceptual dialogue that aims to promote the discussion about public policies for women considering their life cycles, starting from a concept of integral attention to women's health. The review here was based on government documents, books and articles that address the subject of urinary incontinence, public policies, health policies, women's health, and access to the Scielo, Lilacs, PubMed database. It is concluded that the inefficiency of public policies to focus on the health of women, together with the difficulty of the Brazilian health system, corroborate a late diagnosis and difficult access to the treatment of pathology; Elements that contribute to disability in the quality of life of women suffering from urinary incontinence.

KEYWORDS: Female urinary incontinence. Public Health Policy. Quality of Life.

\section{INTRODUÇÃO}

A continência é a capacidade normal de acumular urina na bexiga, com controle consciente sobre o tempo e o lugar para urinar, diferente do que ocorre com os outros animais que urinam por reflexo neuro muscular simples. A micção e a continência urinária são controladas pelos centros neurológicos que integram o sistema nervoso central ao sistema nervoso periférico autônomo e somático garantindo o voluntário ato miccional, bloqueando o reflexo miccional primário (MORENO 2004; MONTEIRO et al., 2007).

A incontinência urinária ocorre quando um dos componentes do processo normal de micção é afetado, alterando o seu funcionamento de forma integrada, desta forma comprometendo tanto a fase de armazenamento como a fase de esvaziamento. Assim, alterações no córtex, no tronco cerebral, na medula espinhal, no Detrusor, ou no complexo esfincteriano podem fazer com que a micção não ocorra de forma satisfatória, gerando uma acomodação irregular da urina e/ou uma eliminação deficitária e incompleta pela bexiga (BAUER; ATALA, 1998), desta forma, diferentes afeções decorrentes do acometimento 
primário do sistema urinário inferior podem estar comprometidos. As duas teorias etiológicas mais aceitas atualmente para a incontinência urinária por esforço em mulheres são a hipermobilidade do colo e/ou uretra, podendo levar a uretrocele ou uretrocistocele e a deficiência do mecanismo esfincteriano intrínseco, com lesão específica do músculo estriado, porém a maioria das mulheres apresenta ambas as causas com proporções diferentes gerando perdas com gravidades específicas (DELORME, 2001).

Os fatores de risco associados à incontinência urinária são: idade; presença de infecção; obesidade devido ao peso sobre o diafragma pélvico; paridade aumentada, principalmente nos casos de parto normais; cirurgias pélvicas que alteram a inervação ou o posicionamento dos órgãos internos; e doenças neurológicas periféricas e centrais. Pacientes com idade avançada, partos normais, partos por fórceps e peso do recém-nascido elevado têm relação direta com a ocorrência da IUE, pois comprometem de forma drástica as estruturas músculo ligamentares do períneo, em contrapartida, partos cesarianos e pacientes uníparas têm riscos significativamente menores quando comparadas com o grupo anterior, por poupar as mesmas estruturas (OLIVEIRA et al., 2008).

As causas exatas que provocam a IU não são claramente estabelecidas, dentre as relatadas que podem interferir com a integridade do assoalho pélvico e do esfíncter urinário são: alterações teciduais na qualidade do colágeno e diminuição da densidade de células musculares lisas na uretra e sua substituição por tecido adiposo e células do tecido conjuntivo, este processo recebe o nome de esclerose uretral (PETROS et al., 1993). Os fatores envolvidos na gênese da IU são múltiplos e podem ter origem no aparelho urinário com lesões vesicais e/ou uretrais, assoalho pélvico com comprometimento do sistema músculo ligamentar com flacidez das fáscias e no sistema neurológico podendo ter origem central ou periférica, além de influências hormonais como o hipogonadismo e psicológicas, e, algumas vezes, origem iatrogênica (RIOS et al., 2010). No quadro é possível identificar com clareza, os tipos de incontinência urinária e suas associações.

As mulheres têm maior probabilidade que os homens de apresentarem perda involuntária de urina, devido à condição anatômica de a uretra ser mais curta e fatores que levam saída da posição intra-abdominal como a gestação, por exemplo, entretanto o número exato de mulheres acometidas pode ser muito maior do que as estimativas atuais. Isso se deve Serv. Soc. \& Saúde, Campinas, SP v.16, n. 2 (24), p. 257-278, jul./dez. 2017 E-ISSN 2446-5992 
ao fato de que a maioria sente vergonha de procurar ajuda, enquanto que outras acreditam que o problema é consequência normal do envelhecimento ou mesmo acreditam que não existe um tratamento (HUNSKAAR et al., 2000). Há também uma variação importante dessa incidência por inexistir uma padronização no tocante ao volume e à frequência da perda (FARRAR et al., 2005).

QUADRO : Classificação dos tipos de incontinência urinária conforme a sintomatologia e a etiologia mais comum

\begin{tabular}{|c|c|c|}
\hline TIPO & SINTOMAS & CAUSAS MAIS COMUM \\
\hline ESFORÇO & $\begin{array}{l}\text { Escape involuntário de urina (quase } \\
\text { sempre em pequenas quantidades) } \\
\text { com aumentos da pressão intra- } \\
\text { abdominal (por exemplo, tosse, } \\
\text { risos ou exercício). }\end{array}$ & $\begin{array}{l}\text { Debilidade e lesão do assoalho pélvico, } \\
\text { que provoca hipermobilidade da base } \\
\text { vesical e da uretra proximal. } \\
\text { Debilidade do esfíncter uretral ou da saída } \\
\text { da bexiga, quase sempre por cirurgia ou } \\
\text { traumatismo. }\end{array}$ \\
\hline URGÊNCIA & $\begin{array}{l}\text { Extravasamento de urina (quase } \\
\text { sempre volumes grandes, ainda que } \\
\text { seja variável) pela incapacidade } \\
\text { para retardar a micção após } \\
\text { perceber a sensação de plenitude } \\
\text { vesical. }\end{array}$ & $\begin{array}{l}\text { Hiperatividade do Detrusor, isolada ou } \\
\text { associada a: } \\
\text { - Condições locais como uretrite, cistite, } \\
\text { tumores, litíase, divertículos; } \\
\text { - Alterações do SNC como AVC, } \\
\text { demência, parkinsonismo, lesão espinhal. }\end{array}$ \\
\hline $\begin{array}{l}\text { TRANSBOR- } \\
\text { DAMENTO }\end{array}$ & $\begin{array}{l}\text { Escape de urina (quase sempre em } \\
\text { pequenas quantidades) secundária a } \\
\text { esforço mecânico sobre a bexiga } \\
\text { distendida ou por outros efeitos da } \\
\text { retenção urinária e a função } \\
\text { esfincteriana. }\end{array}$ & $\begin{array}{l}\text { - Obstrução anatômica: pela próstata ou } \\
\text { por uma cistocele grande; } \\
\text { - Bexiga hipocontratil associada à diabetes } \\
\text { mellitus ou lesão medular. }\end{array}$ \\
\hline FUNCIONAL & $\begin{array}{l}\text { Escape de urina relacionado com a } \\
\text { incapacidade para usar o vaso } \\
\text { sanitário por dano da função } \\
\text { cognitiva ou física; falta de } \\
\text { disposição psicológica ou barreiras } \\
\text { no ambiente. }\end{array}$ & $\begin{array}{l}\text { - Demência grave; } \\
\text { - Imobilidade; } \\
\text { - Ataduras; } \\
\text { - Depressão. }\end{array}$ \\
\hline
\end{tabular}

FONTE: Caderno de Atenção Básica do Ministério da Saúde - ENVELHECIMENTO E SAÚDE DA PESSOA IDOSA (BRASIL, 2006).

A incontinência diminui a qualidade de vida, tornando-a limitada, pois o uso diário de absorventes; o custo com medicações; a frequência miccional aumentada; o odor característico da urina; as restrições de determinadas atividades físicas; e as perdas de urina durante o ato sexual; tudo isso leva ao constrangimento e ao afastamento social, gerando alterações psíquicas, sociais e sexuais. É importante para o médico assistente observar a Serv. Soc. \& Saúde, Campinas, SP v.16, n. 2 (24), p. 257-278, jul./dez. 2017 E-ISSN 2446-5992 
intensidade dos sintomas correlacionando com o nível socioeconômico e cultural da paciente, com o intuito de oferecer com clareza o esclarecimento da sua condição e oferecer o tratamento mais adequado (REIS et al., 2007; GIRÃO et al., 1995).

O termo Qualidade de Vida (QV) possui muitas definições, mas este tem o intuito de avaliar o efeito de determinadas situações que podem influenciar na vida das pessoas. Estão incluídos nesse contexto as condições de saúde física, as funções cognitivas, a satisfação sexual, as atividades do cotidiano, o bem-estar emocional e a vida familiar e social (AUGE et al., 2006). Neste sentido, com todos os prejuízos psicológicos, sociais e econômicos, torna-se necessário a avaliação da qualidade de vida para as pacientes com incontinência urinária assim como o esclarecimento da possibilidade de tratamento da patologia à ser viabilizado pelo sistema de saúde.

\section{INCONTINÊNCIA URINÁRIA FEMININA CONTEXTUALIZADA NAS POLÍTICAS PÚBLICAS DE ATENÇÃO À SAÚDE DAS MULHERES}

A Sociedade Internacional de Continência (ICS) define incontinência urinária (IU) como qualquer perda involuntária de urina, sendo considerado um problema de saúde pública, afetando a qualidade de vida das mulheres (ABRAMS et al,.2002; IUGA, 2009).

A prevalência da incontinência urinária pode variar conforme os tipos de classificação, como IUE, IUU, IUT, IUM e IF. Entre as mulheres, a IUE é a de maior prevalência dentre as incontinências urinárias, varia entre 25 a 35\% na faixa etária entre 45 a 60 anos, e, acima dos 60 anos, as mulheres podem ter probabilidade superior a duas vezes mais perdas urinárias que os homens e, nas pacientes idosas institucionalizadas, essa prevalência pode atingir cerca de 40 a 80\% (OLIVEIRA et al., 2009). Ainda, segundo Higa et al. (2010), com o aumento progressivo da expectativa de vida da população, o número de mulheres na meia idade tende a aumentar cada vez mais, faixa na qual a IUE torna-se mais prevalente. Para Palma et al., (2007), as perdas aos esforços são responsáveis por $49 \%$ das incontinências, variando de 24 a $75 \%$ numa população feminina entre 18 a 90 anos. A segunda causa mais frequente é urge-incontinência, com 30 a $45 \%$ das incontinências, 
particularmente na transição para a menopausa, onde a falta do estímulo estrogênico altera a estabilidade detrusora (POLDEN, et al., 2002).

Estima-se que nos Estados Unidos da América do Norte, segundo a Associação Americana de Urologia, em torno de 33 milhões de mulheres são portadoras de IUE, e 10\% a $30 \%$ destas pacientes apresentam outros tipos de incontinência associados, complicando de forma considerável a qualidade de vida destas pacientes, enquanto a estimativa da Associação Europeia de Urologia é de 22 milhões de mulheres com algum tipo de incontinência urinária (ABRAMS, 2006).

A incontinência urinária gera problemas psicológicos e sociais, sendo estimado que até $25 \%$ dos produtos descartáveis de higiene feminina são usados para amenizar o desconforto das perdas, que muito interfere na qualidade de vida, sendo estimado um gasto anual de 10 a 18 bilhões de dólares para todos os cuidados exigidos no tratamento das incontinências, desde tratamentos fisioterápicos, comportamentais, cirurgias e os tratamentos paliativos como uso de fraldas geriátricas (FONSECA, 2009).

O estudo realizado pela Medical Epidemiologicand Social Aspects of Ageing (MESA) observou que $54 \%$ dos pacientes com perdas urinárias a informavam como queixas clínica ao médico, enquanto $46 \%$ utilizavam métodos de adaptação ao problema para controle urinário, tais como absorventes, micções de horário, restrições hídricas, frequência a lugares com acesso fácil ao banheiro e, também, evitavam viagens longas, sem considerar a perda como grande problema para o seu cotidiano. Com isso, apenas $6 \%$ referiam importante desconforto com as perdas, gerando dificuldade na determinação da prevalência e resultados terapêuticos.

O estudo realizado por Guarisi (2001) observou que das mulheres entre os 30 e 45 anos de idade, a prevalência chegava a aproximadamente, $18 \%$. No caso das mulheres que estavam no climatério, até $35 \%$ apresentavam queixas de incontinência urinária de esforço, embora possa ser uma avaliação sub diagnosticada, uma vez que parte das mulheres não relata a perda de urina aos seus médicos clínicos assistentes e mesmo para os ginecologistas por constrangimento ou até por considerarem um processo natural do envelhecimento ou decorrente das gestações (AUGE et al., 2006).

A incontinência urinária feminina, na atualidade, tem levantado muito interesse dos profissionais que atuam nessa área, dentre eles citamos os urologistas, os ginecologistas, geriatras e fisioterapeutas, em decorrência dos múltiplos fatores e consequências relacionadas Serv. Soc. \& Saúde, Campinas, SP v.16, n. 2 (24), p. 257-278, jul./dez. 2017 E-ISSN 2446-5992 
à doença. Assim têm se procurado investigar a qualidade de vida das mulheres incontinentes, diante das transformações que acarretam o dia-a-dia de forma ampla, comprometendo o desempenho no trabalho, no cotidiano doméstico e nas relações afetivas e sexuais (VOLKMER et al., 2012). No domínio da sexualidade feminina, o impacto negativo pode ser de até $60 \%$ das pacientes com algum tipo de incontinência, sendo grave em $36 \%$ dos casos, podendo ocasionar até a depressão (PALMA et al., 2007).

A história natural da incontinência urinária tende a ser progressiva, com perdas geradas por menores esforços com o passar do tempo. A perda de qualquer função fisiológica, como a capacidade de armazenamento e esvaziamento vesical adequado, mesmo que temporária, é limitante e afeta aspectos importantes na secularidade diária dessas mulheres. Formosa et al.,(1994) relataram o enrijecimento uretral associado à epitelização deficitária da mucosa e perda do coxim vascular, além da perda da posição anatômica do complexo uretrovesical, e correlacionaram com o hipogonadismo e o histórico gestacional com a ocorrência da IUE.

Porém, parece claro pelos estudos populacionais que, embora haja correlação, esses não são os únicos fatores determinantes, visto que $17 \%$ das mulheres incontinentes eram uníparas e $25 \%$ da amostra tinham idade abaixo de 35 anos. Todos os fatores relacionados aos aumentos súbitos e recorrentes da pressão intra-abdominal associado ao excesso de peso sobre o diafragma urogenital podem associar a gênese das incontinências. No Brasil, os estudos epidemiológicos sobre a incontinência urinária em mulheres, podem ser subestimados (BARACHO, 2007).

O grande desafio brasileiro para o século XXI é oferecer suporte de qualidade de vida para uma população com mais de 32 milhões de idosos, quadro este que se assemelha aos países desenvolvidos, sendo que a maioria desses idosos é do sexo feminino, constituindo o segmento populacional que mais cresceu nos últimos anos, considerando que $12,8 \%$ da população são constituídas por idosas, isso se deve em decorrência da melhora da qualidade geral de vida e da oferta à Atenção Básica elementar de saúde. O envelhecimento, antes considerado um fenômeno, hoje, faz parte da realidade da sociedade, sendo ainda que na maioria dos idosos a constatação do nível socioeconômico e educacional é baixo e com alta prevalência de doenças crônicas e incapacitantes (BRASIL, Ministério da Saúde, 2004). 
A garantia da atenção à saúde das mulheres no Sistema de Saúde brasileiro, que é um dos maiores do mundo e que assumiu essa obrigação com a promulgação da Constituição de1988, tornou-se factível quando foi definido nas Leis 8080/90 e 8142/90, como

[...] o conjunto de ações e serviços públicos de saúde, prestados por órgãos e instituições públicas federais, estaduais e municipais, da administração direta e indireta e das fundações mantidas pelo poder público, que se propõe a garantir a atenção integral e gratuita para toda a população, desde o atendimento aos processos de baixa complexidade até os que demandam de alta tecnologia (GIOVANELLA et al., 2009, p. 112).

Quando a oferta de serviços públicos não for suficiente para garantir a assistência à população de uma determinada região, quer município, quer estado, o SUS, através dos seus gestores, poderá contratar os serviços prestados pela iniciativa privada, através de licitação pública gerando então os convênios e os contratos, com controle e fiscalização periódicos. Os recentes avanços legais do SUS, explicitados no decreto 7508/11 e através das Leis 12.466/11 e 141/12, levaram reais ampliações para a universalização e qualificação da Atenção Básica à saúde, com isso tenta-se resolver 85 a 90\% das necessidades de saúde da população (GIOVANELLA et al., 2009), aumentando assim, as chances de melhor atendimento às mulheres que não têm acesso ao serviço privado custeado de forma particular ou por plano de saúde.

Nesse sentido, é necessário promover ações que melhorem o acesso a pacientes com incontinência urinária para a promoção da Universalidade, Integralidade e Equidade:

1) Universalidade de acesso em todos os níveis de assistência onde todos têm o mesmo direito de obter as ações e os serviços de que necessitam independentemente de complexidade, custo e natureza dos serviços envolvidos. Com a universalidade, as condições socioeconômicas da população e a inserção no mercado de trabalho não devem implicar acesso diferenciado a determinados tipos de serviços, pois os riscos de adoecimento e financiamento passam a ser repartidos de forma solidária, sendo de responsabilidade de toda a sociedade;

2) Equidade sendo entendida como a igualdade na assistência à saúde, sem preconceitos ou privilégios de qualquer espécie, sem discriminação no acesso aos serviços de saúde, ou seja, não é aceitável que somente alguns grupos, por motivos relacionados à renda, cor, gênero ou religião, tenham acesso a determinados serviços e outros não, exceto em necessidades diferenciadas de saúde, sendo essas orientadas tecnicamente pelo SUS;

3) Integralidade da assistência onde se entende como o conjunto articulado e contínuo de ações e serviços preventivos e curativos, individuais e coletivos, exigidos para cada caso em todos os níveis de complexidade do sistema. Estas ações devem ser associadas às assistências ambulatoriais e hospitalares voltadas para o diagnóstico, $\mathrm{o}$

Serv. Soc. \& Saúde, Campinas, SP v.16, n. 2 (24), p. 257-278, jul./dez. 2017 E-ISSN 2446-5992 
tratamento e a reabilitação. Também à articulação necessária entre as políticas no sentido de atuar sobre o processo de saúde e doença, garantir as condições satisfatórias de saúde da população (PISANESCHI, 2009, p. 68).

No entanto, para que os princípios do SUS seja uma realidade é necessária à participação efetiva da comunidade, pois essa é a garantia de que a população, por intermédio de suas entidades representativas como a participação efetiva nos Conselhos de Saúde, possa participar do processo de construção de diretrizes e prioridades para a política de saúde, objetivando características peculiares de cada região, de fiscalização do cumprimento das leis e normas do SUS e de controle e avaliação dos serviços prestados cobrando acima de tudo qualidade no atendimento. Essa participação ocorre pela realização das Conferências de Saúde, onde todos os Conselhos de Saúde se reúnem para então se tornarem um canal permanente de diálogo e interação entre os gestores, os profissionais de saúde e a população (GIOVANELLA et al., 2009).

Considerando que a incontinência urinária afeta mulheres de todas as classes sociais, distribuída em todos os municípios indiscriminadamente, torna-se necessário que a descentralização administrativa promova maior autonomia e responsabilidade aos municípios em decidir e implantar ações e serviços de saúde, incluindo aqui os serviços de atenção à mulher com incontinência urinária. Dessa forma, para que não haja ociosidade na oferta de procedimentos, sendo esta a condição que se encontra os encaminhamentos para a realização do estudo urodinâmico, se faz necessário organizar uma rede de serviços que ofereçam tecnologias de diferentes níveis de especialização, complexidade e custo. Nesse caso, é preciso definir os serviços por níveis de atenção com "hierarquização", e distribuí-los geograficamente - "regionalização" (GIOVANELLA et al., 2009).

O desafio para a Atenção Básica na resolução dos problemas de saúde das mulheres com incontinência urinária está no fortalecimento da integração da rede e na garantia de acesso à atenção especializada, passa por este viés a necessidade de informação, conscientização e capacitação dos médicos generalistas associado à presença do especialista para dar o suporte necessário para auxílio no diagnóstico e tratamento nos casos mais complexos. No entanto, deve-se evitar a presença de solução de continuidade do sistema decorrente dos diferentes prestadores de saúde municipal, estadual e privados contratados. A diversidade de prestadores de serviços pode dificultar uma regulação pública capaz de construir um sistema de atenção integral, porém torna-se necessário toda vez que o Estado não é capaz de suprir essa demanda, e a continuidade desses cuidados é considerada como importante indicador para a avaliação do desempenho do sistema de saúde (VIACAVA, 2004). 
Essas mulheres estão legalmente protegidas pelo Plano Nacional de Políticas Públicas para as Mulheres (PNSPM - 2005), que propõe promover a melhoria da saúde das mulheres brasileiras mediante a garantia de direitos constituídos, e ampliar o acesso aos meios e serviços de promoção, prevenção, assistência e recuperação da saúde, em todo o território brasileiro; contribuir para a redução da morbidade e mortalidade feminina no Brasil, por causas evitáveis, em todos os ciclos de vida, sendo que conforme se observa na incidência e na prevalência das incontinências urinárias existe um período específico na vida das mulheres onde a fremência deste agravo é mais presente, ou seja, no climatério, os diversos grupos populacionais, sem qualquer forma de discriminação; e ampliar, qualificar e humanizar a atenção integral à saúde da mulher no Sistema Único de Saúde, implantando, com as equipes do Programa Saúde da Família (PSF), nos municípios, a atenção qualificada às mulheres com queixas clínico-ginecológicas (BRASIL, 2004).

Apesar de todo o contexto das políticas públicas específico paras as mulheres, as pacientes com incontinência urinária, nesse sistema, podem estar enfrentando muitos desafios em relação ao acesso entre a Atenção Primária e outros níveis assistenciais mais especializados como: dificuldade em se realizar referência e contra referências dessas pacientes com demora na resolução de suas demandas; inexistência de um sistema logístico que resulta em falta de mecanismos de regulação para a marcação de consultas e exames com especialistas; pouca comunicação entre os profissionais de saúde de níveis assistenciais distintos, muitas vezes por falha na formação dos médicos generalista, outras vezes por carência de médicos especialistas, além de um prontuário único integrado a todo o sistema que permita o acesso à conduta e aos registros clínicos das pacientes (MENDES, 2009).

A formulação da Política Nacional de Atenção Integral à Saúde da Mulher (PNAISM, BRASIL, 2004) teve como foco a preocupação em reduzir os índices de morbidade e de mortalidade por causas previsíveis e evitáveis das mulheres nas várias faixas etárias. Com isso, áreas importantes que anteriormente não eram contempladas, dentre elas, a preocupação com o climatério, menopausas e queixas ginecológicas, passaram a ser discutidas e assistidas, evoluindo assim a abrangência das políticas públicas para uma visão que vai além da abordagem materno-infantil para uma atenção mais completa da mulher (FREITAS, 2009).

Considerando o cotidiano da mulher com incontinência urinária, a PNAISM reforça a humanização da atenção em saúde, regulamentando que a humanização e a qualidade da atenção são aspectos primordiais. Nesse sentido, humanizar é muito mais que tratar bem ou de forma amigável, porém devem ser consideradas questões de acessibilidade ao serviço nos três níveis da assistência (federal, estadual e municipal), provisão de tecnologias necessárias, formulação de referência e contra referências, com isso proporcionar a usuária do sistema público condições necessárias para o seu 
acolhimento, gerando assim uma solução para o diagnóstico, tratamento e restabelecimento precoce. (GIOVANELLA et al., 2009).

Tratando-se especificamente da incontinência urinária não há qualquer protocolo deste agravo nas políticas públicas para as mulheres, mesmo quando se aborda o climatério. Entretanto, há uma proposta com protocolo específico para as incontinências urinárias na política para os idosos, porém sem discriminação de gênero, o que se torna um grande problema visto que a origem etiológica pode diferir entre homens e mulheres incontinentes.

A Política Nacional de Saúde da Pessoa Idosa (PNSPI, BRASIL, 2006) foi recentemente atualizada, considerando o pacto pela saúde e suas diretrizes operacionais para consolidação do SUS, entendendo que o processo de envelhecimento apresenta vertentes particulares, caracterizado por doenças e/o condições crônicas não transmissíveis, porém com possibilidade factível para a prevenção, controle e tratamento, e por limitações incapacitantes que podem ser amenizadas. Dentre tais desafios ressalta-se a escassez de equipes multiprofissionais e interdisciplinares com conhecimento em envelhecimento e saúde da pessoa idosa, principalmente na Atenção Básica que deveria resolver à grande maioria das demandas geradas no contexto da saúde da população (MARTINS, 2000).

Nesse direcionamento, a Política Nacional do Idoso em seu Caderno de Atenção Básica, com o tópico "Envelhecimento e Saúde da Pessoa Idosa", destina um capítulo completo para avaliação, acompanhamento e direcionamento do paciente com incontinência urinária, no intuito de aperfeiçoar o tratamento desses pacientes. Neste capítulo, abordam as causas agudas, como as endocrinológicas (Diabetes e Hipogonadismo); causas psicológicas (Depressão, Ideias Delirantes); causas farmacológicas (diuréticos anti-hipertensivos, etc.); causas infecciosas; causas neurológicas (AVC, Parkinson, Delírio); e causas crônicas, como a incontinência urinária; oferecendo um protocolo de diagnóstico e tratamento a ser seguido pelo médico generalista antes de encaminhar para o especialista (BRASIL, 1990).

\section{CONTRAPONDO A QUALIDADE DE VIDA DAS MULHERES COM INCONTINÊNCIA URINÁRIA E POLÍTICA PÚBLICA DE SAÚDE PARA MULHERES}

Com o objetivo de diminuir a diferença de atenção às pessoas de acordo com o gênero, o governo federal criou em janeiro de 2003, com status de Ministério, a Secretaria Especial de Políticas para as Mulheres da Presidência da República (SPM/RP). O primeiro Serv. Soc. \& Saúde, Campinas, SP v.16, n. 2 (24), p. 257-278, jul./dez. 2017 E-ISSN 2446-5992 
grande passo foi à realização da I Conferência Nacional de Políticas para as Mulheres (ICNPM), em julho de 2004, sendo proposta a elaboração do Plano Nacional de Políticas para as Mulheres - (PNPM) (BRASIL, 2004).

O PNPM reconhece o papel fundamental do Estado, que através de ações e políticas públicas de combate à desigualdade de gênero, tem aumentado a possibilidade de atuação em diversas áreas, atendendo diretamente às necessidades das mulheres em toda a sua diversidade e no seu dia-a-dia (BRASIL, 2004).

Esse plano deveria ser implantado em todas as esferas do poder municipal, estadual e federal, trabalhando junto com a sociedade para sua execução. Considerando que o acesso e participação das mulheres nos espaços de poder é um instrumento essencial para democratizar o Estado e a sociedade, a participação popular através dos Conselhos de Saúde se torna primordial para que o tema seja conhecido e discutido, quando se aborda a saúde da mulher. O PNPM tem várias ações, distribuídas em prioridades, que foram definidas a partir dos debates estabelecidos na ICNPM. Foram traçadas quatro linhas de atuação, consideradas como as mais importantes e urgentes para garantir, de fato, o direito a uma vida melhor e mais digna para todas as mulheres (BRASIL, 2004).

As linhas foram assim divididas:

1) Autonomia, Igualdade no mundo do Trabalho e Cidadania - atuando nas áreas econômicas e financeiras, no trabalho social e na política, e gerando acesso a bens e serviços, à cidadania, a terra e à moradia;

2) Educação Inclusiva e Não-Sexista - incorporar à perspectiva do gênero na educação, não discriminatório, com acesso à educação básica para as mulheres jovens e adultas, com visibilidade da contribuição das mulheres na história da humanidade. Ampliar o acesso à educação infantil (creches e préescolas) e promover a alfabetização;

3) Saúde das Mulheres, Direitos sexuais e Direitos Reprodutivos promover a prevenção, assistência e recuperação da saúde com ampliação do acesso aos meios de serviços; garantir direitos sexuais e reprodutivos; trabalhar para reduzir a mortalidade feminina de causas evitáveis; ampliar, qualificar e humanizar a atenção integral à saúde da mulher no Sistema Único de Saúde através da saúde da família, dos centros de atenção psicossocial; ampliar ações de planejamento familiar; e promover a prevenção e controle das doenças sexualmente transmissíveis;

4) Enfrentamento à Violência contra as Mulheres - implantar uma Política Nacional de Enfrentamento à Violência contra a Mulher, garantido um atendimento integral, humanizado e de qualidade, instituir as redes de atendimento às mulheres em situação de violência em todos os estados 
brasileiros, ampliar o acesso à justiça e à assistência jurídica gratuita (BRASIL, 2004, p. 74).

Caminhando com uma visão mais ampla sobre a categoria gênero, o Ministério da Saúde elaborou a Política Nacional de Atenção Integral à Saúde da Mulher (PNAISAM) com o compromisso de implantação de ações de saúde que contribuam para a garantia de direitos as mulheres e para reduzir a morbidade por causas previsíveis e evitáveis, reforçar a integralidade e a promoção da saúde (BRASIL, 2004).

Dentro dessa política existe um tópico importante com o título "Humanização e Qualidade: Princípios para uma Política de Atenção Integral à Saúde da Mulher”, em que se observa que a humanização e a qualidade na atenção em saúde são condições essenciais para que as ações de saúde se traduzam na resolução dos problemas identificados, na satisfação das usuárias dando dignidade e hombridade as pacientes, aos servidores e aos gestores públicos (BRASIL, 2004).

As diretrizes da PNAISM são (BRASIL, 2004):

1) O SUS deverá estar orientado e capacitado para a atenção integral, contemplando a promoção e as necessidades de saúde e o controle das patologias prevalentes, com garantia do direito à saúde;

2) A PNAISM deverá atingir as mulheres em todos os ciclos da vida, grupos populacionais específicos e resguardar as especificidades das diferentes faixas etárias;

3) A elaboração, a execução e a avaliação das políticas de saúde da mulher com o objetivo de alcançar todos os aspectos da saúde sexual e reprodutiva, e nortear-se pela perspectiva de gênero, de raça e etnia. Sendo a participação da sociedade civil fundamental nesta área;

4) A gestão desta política deverá estabelecer dinâmica inclusiva para atender às demandas antigas e às novas demandas elencadas pela população feminina;

5) As práticas em saúde deverão nortear-se pelo princípio da humanização, demonstrando o interesse em resolver problemas e diminuir o sofrimento assolado ao processo de adoecimento e morte da clientela feminina e seus familiares (BRASIL, 2004, p. 147).

Dentro dos objetivos específicos e estratégias da PNAISM, quanto à incontinência urinária, caberia ressaltar os seguintes tópicos (BRASIL, 2004):

1) Ampliar e qualificar a atenção clínico-ginecológica, fortalecendo a atenção básica no cuidado com a mulher, ampliando o acesso e qualificando a atenção clínico-ginecológico na rede SUS;

2) Promover à atenção obstétrica e neonatal, qualificada e humanizada, com o intuito de reduzir lesões perineais evitáveis; 
3) Implantar e programar a atenção à saúde da mulher no climatério na rede SUS, visando à atenção à saúde da mulher na terceira idade, incluindo a abordagem de gênero na Atenção à Saúde do Idoso, relacionando as alterações hormonais geradas por esta fase da vida (BRASIL, 2004, p. 86).

O termo qualidade de vida (QV) como critério de avaliação, tem por objetivo classificar as condições de saúde física, funções cognitivas e mentais, satisfação sexual, atividades do cotidiano, o bem estar emocional isolado e na vida familiar e social. A perda de qualquer função fisiológica, como a capacidade de enchimento e esvaziamento vesical adequada, mesmo que temporária, é limitante e afeta aspectos sociais e psicológicos das pacientes, gerando grande transtorno isolado ou coletivo/familiar. Por isso, a Sociedade Internacional de Continência (ICS) tem recomendado a inclusão de um questionário de qualidade de vida em todo e qualquer estudo sobre a incontinência urinária (FONSECA, et al., 2005; AUGE, et al., 2006).

A Organização Mundial de Saúde (OMS) iniciou um projeto de avaliação internacional de qualidade de vida, devido à relevância do tema e suas implicações sociais, levando em consideração decisões de tratamento, utilização de novos fármacos e políticas públicas e de pesquisa. A maior parte dos instrumentos de medida de qualidade de vida é baseada em questionários, quer estes sejam usados com aplicações gerais quer com especificidades para cada agravo investigado ou referente a aspectos singulares do cotidiano analisado, estes avaliam os diversos aspectos e dimensões da vida dos pacientes, como o físico, o psicológico, o social, o desempenho geral, a dor, o sono, e os sintomas específicos de cada doença. No caso das incontinências urinárias existem vários questionários específicos e também com investigação da vida sexual associada (FONSECA, et al., 2005).

Considerando que, culturalmente e principalmente nas pacientes com nível socioeconômico e cultural mais desfavorável, a ideia concebida de que a incontinência urinária faz parte do envelhecimento, existe uma associação com a não procura para melhor entendimento das causas e possível tratamento do evento. Como consequência, o tratamento que deveria ser feito nas idades menos avançadas, com resultado mais duradouro e com menor risco de complicações, em função do menor número de co-morbidades, não ocorre no momento adequado, associado ao desconhecimento das pacientes, está o despreparo dos médicos generalista em abordar e investigar esta patologia (AUGE et al., 2006; VOLKMER, 2012). 
A vida das mulheres incontinentes é afetada de diversas maneiras. Passam a se preocupar com disponibilidade de banheiros principalmente nos casos de perdas por urgência, envergonham-se com o odor de urina e sentem-se frequentemente sujas, chegando a apresentar lesões cutâneas como dermatites amoniacais e infecções urinárias repetidas, muitas com dificuldade nas relações sexuais por dor local ou por vergonha ao se apresentarem aos parceiros, perdas urinárias durante o sono e, consequente, aumento do cansaço, podendo desencadear quadros de depressão e ansiedade, intensificados pelas sensações de baixa autoestima e gerando também incômodo familiar que no início toleram, porém com o passar do tempo começam a esboçar repulsa e repreensão, prejuízo nas atividades diárias, no trabalho, em casa e nas atividades físicas (VOLKMER, 2012, AUGE et al., 2006).

As portadoras de incontinência urinária, com frequência, não procuram o profissional de saúde com a queixa principal de perda de urina, mesmo sendo o seu ginecologista. Muitas vezes, considera uma evolução natural do envelhecimento e da gravidez múltipla, o que se observa é que falta informação geral sobre esta patologia, tanto para as pacientes como para as pessoas que trabalham com a saúde. É a severidade da perda que desencadeará o desejo para o diagnóstico e tratamento adequado. O fato é que mais de $70 \%$ das pacientes sofrem por constrangimentos gerando prejuízos psíquicos importantes, até que rompam com a vergonha e apresentem de forma clara o seu problema (AUGE et al., 2006, VOLKMER, 2012).

De acordo com Volkmer, 2012 e Fonseca et al., 2005, os resultados gerados por questionários de qualidade de vida para as pacientes com IUE mostraram inúmeras dificuldades em suas experiências de vida:

1) Dificuldades encontradas para lidar com a IU: sentimentos negativos, como vergonha, sensação de voltar à infância e medo de exalar cheiro de urina; subestimação do problema; concepção sobre as causas da IU com ideias preconcebidas a respeito, como envelhecimento, o número de gestações e partos em idade precoce; acesso ao cuidado profissional, principalmente especialista no assunto; diferenças culturais; limitações causadas pela IU;

2) Modos de enfrentamento: autogestão da IU, manobras para esconder as perdas; busca do apoio dos profissionais naquelas pacientes em que as perdas eram mais acentuadas; pensamentos positivos para submeter-se aos 
tratamentos propostos; processo de vitimização em que as IU eram apenas mais um dos muitos problemas;

Os estudos mostraram a distância entre a vivência das mulheres incontinentes em relação à família e à sociedade, pois elas preferem enfrentar o problema sozinhas e, muitas vezes, adotando a tática do silêncio, com isso criam estratégias para amenizar o sofrimento, as perdas, como diminuição da injeção de líquidos, uso de absorventes e escolha seletiva de local com banheiros (VOLKMER, 2012).

Tanto o Plano Nacional da Política Pública do Idoso como o Plano Nacional da Política Pública para as Mulheres demonstraram uma grande preocupação com a qualidade de vida e humanização.

A humanização como política que abarca toda a rede SUS visa traduzir os princípios dos postulados de modo que possa ser aplicado para construir trocas solidárias e comprometidas com a dupla tarefa de produção de saúde dos usuários e produção com valorização de sujeitos envolvidos na prestação dos serviços, com o aumento do grau de corresponsabilidade na produção da saúde, tendo como foco as necessidades dos cidadãos, com a melhoria nas condições de trabalho, com a formação de rede de equipes multidisciplinares e transdisciplinares nos vários níveis da gestão do SUS (federal, estadual e municipal) e com o intuito de aperfeiçoar o acesso ao exame mais sofisticado e/ou ao especialista (BRASIL, 2004).

A PNAISM reforça a humanização da atenção em saúde, concebendo que a humanização e qualidade da atenção são aspectos indissociáveis, devendo ser consideradas as questões de acessibilidade ao serviço nos três níveis da assistência, provisão de insumos e tecnologias necessárias, formulação de sistemas de referência e contra referência (BRASIL, 2004). Nesse sentido, se enquadra a realização do estudo urodinâmico, como propedêutica bem indicada para facilitar diagnóstico e programar tratamentos, não sendo concebível a espera em longas filas até que se consiga fazer este exame.

\section{CONSIDERAÇÕES FINAIS}

A incontinência urinária acaba se caracterizando por uma doença que acomete grande parte dos pacientes idosos, principalmente as mulheres. Portanto, para um acolhimento humanizado dessas mulheres, os profissionais de saúde devem compreender as Serv. Soc. \& Saúde, Campinas, SP v.16, n. 2 (24), p. 257-278, jul./dez. 2017 E-ISSN 2446-5992 
especificidades dessa população e a própria legislação brasileira vigente. Para isso, devem estar preparados para lidar com as questões do processo de envelhecimento, sabendo diferenciar a questão do gênero nesta fase da vida no que concerne à dimensão subjetiva do sexo feminino e romper com a precariedade nas equipes multidisciplinares, pois é preciso reconhecer a integração entre a rede básica e o sistema de referências para facilitar o acesso dessas mulheres aos diversos níveis de complexidade da atenção (BRASIL, 2004).

Buscando melhorar a qualidade de vida das pacientes e reduzir o encaminhamento indevido para a realização do exame urodinâmico, é recomendado a avaliação das disfunções vesicais por meio de um método propedêutico que permite a avaliação funcional do trato urinário inferior, através da história clínica, exame físico detalhado e aplicação de questionário como auxílio no diagnóstico (DONG et al., 2006).

A avaliação do impacto da incontinência urinária e das intervenções a ela relacionadas pode ser feita de forma objetiva ou subjetiva com a utilização de questionário padronizado, ou por entrevista, a fim de que seja avaliado o impacto da perda urinária na vida dos pacientes e qual a melhor forma de abordagem propedêutica e terapêutica (KUBLIK et al., 2004; SIMÃO, 2001).

Entretanto, o que se observa é que o direito à saúde está longe de ser uma realidade. $\mathrm{O}$ Estado não investe o necessário não suprindo assim as demandas elencadas pela população, os trabalhadores da saúde não são remunerados satisfatoriamente e muitos escolhem por trabalhar na Medicina privada, principalmente os médicos, os gestores não contam com os recursos e equipamentos que deveriam, pois há uma burocracia que emperra toda a possibilidade e rapidez necessária, e a população não participam do controle sobre a gestão das políticas para reivindicar os seus direitos, por desconhecimento deste direito ou por omissão em não participar dos Conselhos de Saúde. A promoção e humanização da saúde são processos longos e deveriam ser dinâmicos (SILVA et al., 2007).

As práticas em saúde, então, deverão nortear as atitudes e comportamentos dos profissionais da área a fim de que contribuam para reforçar a atenção à saúde como direito constitucional, que melhorem o grau de informação das mulheres em relação ao seu corpo e suas condições de saúde, passando então pelo víeis de profissionais capacitados, responsáveis por orientar e divulgar os agravos que mais acometem a sua região, como prevenir e buscar Serv. Soc. \& Saúde, Campinas, SP v.16, n. 2 (24), p. 257-278, jul./dez. 2017 E-ISSN 2446-5992 
estratégias e possibilidade de tratamento com o uso de tecnologias apropriadas a cada caso e com o objetivo de resolver problemas, diminuindo o sofrimento dessa população (TAVARES et al., 2009).

A nova visão sobre a mulher deve passar pelo entendimento de sua integralidade, deixando de ser apenas mama, colo uterino e gestação. Mais do que isso, é necessário atuar sobre os diferentes ciclos da vida da mulher, compreendendo a infância, adolescência, idade adulta e senectude, dentro de uma perspectiva de direito a qualidade de vida e opção quanto ao que fazer consigo mesma (MEDEIROS et al., 2005).

Tendo em vista que o Sistema de Saúde apresenta dificuldades em assistir à mulher em diversas áreas, como por exemplo, o pré-climatério e o climatério, sendo esta a faixa etária em que está a grande maioria das mulheres que apresentam o quadro de incontinência urinária, torna-se essencial questionar a real implantação das PNAISM nos municípios e Estados brasileiros. As políticas de saúde ainda não contemplam as especificidades da saúde da mulher de meia idade, tendo em vista as intensas transformações potencializadas pela menopausa e pelo inevitável processo de envelhecimento. As necessidades de cada fase do ciclo vital apresentam características próprias e a suspensão imediata dos hormônios femininos gera transformações rápidas e muitas previsíveis ao corpo da mulher (MARGIS et al., 2001).

Recebido em 03.03.2017 - Aprovado em 26.12.2017

\section{REFERÊNCIAS}

ABRAMS, P. Urodynamics. 3ed.Springer, 2006.

ABRAMS, P.; CARDOSO, L.; FALLl, M. The standardization of terminology of lower Urinary Tract Function: report fron the standardization sub-committee of the International Continence Society. Neurourol Urodyn, v. 21,p. 167-178, 2002.

AUGE, A.P. et al. Comparação entre os índices de qualidade de vida em mulheres com incontinência urinária submetidas, ou não, ao tratamento cirúrgico. Revista Brasileira de Ginecologia e Obstetrícia, v.28, n. 6,p. 352-357, jun.2006.

BARACHO, E. Fisioterapia aplicada à obstetrícia, urogenicologia e aspectos de mastologia. 4. ed. Rio de Janeiro: Guanabara Koogan, 2007. 
BAUER, S.B.; ATALA, A. Bladder dysfunction. In: BARRAT, T. M.; AVNER, E. D.; HARMON, W. E. (Editors). Pediatric Nephrology. 4thed. Baltimore: Lippincott, Willians\& Wilkins, 1998. p. 913-931.

\section{BRASIL. Constituição (1988). Constituição [da] República Federativa do}

Brasil. Brasília: Senado Federal, 1988.

BRASIL. Lei n. 8.080, de 19 de setembro de 1990: [Lei Orgânica da Saúde]. Dispõe sobre as condições para a promoção, proteção e recuperação da saúde, a organização e o funcionamento dos serviços correspondentes e dá outras providências. Diário Oficial [da] República Federativa do Brasil, Brasília, DF, 20 set. 1990. p. 18.055.

BRASIL. Ministério da Saúde. Secretaria-Executiva. Qualisus Política de qualificação da atenção à saúde. Brasília, DF, 2004.

BRASIL. Ministério da Saúde. Caderno de Atenção Básica - Envelhecimento e saúde da pessoa idosa. Brasil, 2006.

BRASIL. Ministério da Saúde. Síntese das diretrizes para a política de atenção integral à saúde da mulher 2004 a 2007. In: Ministério da Saúde (Org.). 2004: ano da mulher. Brasília: Ministério da Saúde, 2004. p. 77-82. (Série E. Legislação de saúde).

BRASIL. Ministério da Saúde (Org.). 2004: ano da mulher. Brasília: Ministério da Saúde; 2004. (Série E. Legislação de saúde).

DELORME, E. La bandellettetrans-obturatrice: um procede miniinvasipourtraiterI'incontinceurinaire d’effort de Lafemme. Progrès em Urologie, v. 1, p. 1306-1313, 2001.

DONG, D.; XU, Z.; SHI, B.; CHEN, J.; JIANG, X.; WANG, H. Urodynamic study in the neurogenic bladder dysfunction caused by intervertebral disk hernia. NeurourolUrodyn, $v$. 25, n. 5, p. 446-450, 2006.

FARRAR, D. J.; WHITESIDE, C. J.; OSBORNE, J. L.A urodynamic analysis of micturition symptoms in the female. Surg. Gynec. Obstet., v. 141, p. 875, 2005.

FONSECA, E.S.M.; CAMARGO, A.L.M. et al. Validation of a quality of life questionnaire (King's Health Questionnaire) in Brazilian women with urinary incontinence (2005).Disponível em: <http://www.scielo.br>.Acesso em: 28 abril 2009. FORMOSA, A. M.; BRINCAT, M. P.; CARDOZO, L. D. Collagen. In: LOBO, R. A. (Eds) Treatment of postmenopausal women. New York: Raven Press, 1994. 
FREITAS, G. L. Discutindo a Política Pública de Atenção a Saúde da Mulher no contexto da promoção a Saúde. Revista Eletrônica de Enfermagem, v. 11, p. 424-8, nov. 2009. GIOVANELLA, L.; MENDONÇA, M. H.; ALMEIDA, P. F.; ESCOREL, S.; SENNA, M. C. M.; FAUSTO, M.C.R.; DELGADO, M.M.; ANDRADE, C.L.T.; CUNHA, M.S.; MARTINS, M. I. C.; TEIXEIRA, C.P. Saúde da família: limites e possibilidades para uma abordagem integral de antenção primária à saúde no Brasil. Revista Ciência e Saúde Coletiva, v. 14, n. 3, p. 783-794, 2009.

GUARISI, T.; AAR, O.; PINTO - NETO, M. J. M. O.; ORCESI, A.; COSTA - PAIVA, L.H.; FANDES, A. The Searchof Medical CarebyWomenwithUniraryIncontinece. Rev. Bras. Ginec. Obst. v.23, n. 7, p. $439-443,2001$.

HIGA, R.; RIVORÊDO, C.R.S.F.; CAMPOS, L.K.; LOPES, M.H.B.M.; TURATO, E.R. Vivências das mulheres brasileiras com incontinência urinária. Texto Contexto Enfermagem, v. 19, n. 4, p. 627-635, out./dez. 2010.

HUNSKAAR S, ARNOLD E. P., BURGIO K., DIOKNO A. C., HERZOG A.R., MALLETT V.T. Epidemiology and natural history of urinary incontinence. International Urogynecology Journal and Pelvic Floor Dysfunct. v.11, n.5, p. 301-19, 2000.

IUGA Int. Urogynecol J, supplement 2, 2009.

KUBLIK, K.; BLACKWELL, L.; HEIT, M. Does socioeconomic status explainracial difference in urinary incontinence knowledge? Am J Obstet/ gynecol. v. 191, n. 1, p. 188193,Jul. 2004.

MARGIS, R.; CORDIOLI, A.V. Idade adulta: meia idade, ciclo da vida humana; uma perspectiva psicodinâmica. Porto Alegre. Artmed Editoria,2001. p.159-67.

MARTINS, F.G. Tratamento conservador da incontinência urinária feminina, Urologia

Contemporânea - órgão oficial do departamento de urologia da Associação Paulista de Medicina, v. 6, n. 1, 2000.

MEDEIROS, V.C.R.D.; MEDEIROS, R.C.; MORAES, L.M.; MENEZES FILHO, J.B.; RAMOS, E.S.N.; SATURNINO, A.C.R.D. Câncer de colo de útero; análise epidemiológica e citopatologica no Estado do Rio Grande do Norte. RBAC, v. 37, n.4, p. 227-231, 2005 MENDES, E.V. O SUS e a Atenção Primaria à Saúde. Revista Atenção à Saúde. Dez. 2009. 
MONTEIRO, M.V.C.; SILVA, FILHO, A.L. Incontinência urinária. In: BARACHO, E.

Fisioterapia aplicada à obstetrícia, uroginecologia e aspectos de mastologia. 4. ed. Rio de Janeiro: Guanabara Koogan, 2007. p. 280-90.

MORENO, A.L. Fisioterapia em uroginecologia. 1 ed. São Paulo: Manole, 2004.MORI M.E. Sistema Único de Saúde e políticas públicas: atendimento psicológico à mulher menopausada no Distrito Federal, Brasil. Cadernos Saúde Pública, Rio de Janeiro, v. 22, n. 9, p. 1825833, set. 2006.

OLIVEIRA, C.; LOPES, M.A.B. Efeitos da cinesioterapia no assoalho pélvico durante o ciclo gravídico-puerperal. Disponível em: 〈http://www.teses.usp.br>. Acesso em: 25 abril 2008 .

OLIVEIRA, M. S.; TAMANINI, J. T. N; CAVALCANE, G. A. Validación of the prolapse quality-of-life questionnaire (P-QOL) in portuguese versión in Brazilian women.

International Urogynecology Journal, v. 20, n. 10, p. 1191-1122, 2009.

PALMA, P.C.R.; RICCETTI,C.; FRAGA,R.; PORTUGAL,S.; DAMBROS,M.; RINCÓN, M.E.; SILVEIRA, A.; NETTO JR., N.R. Anatomia Tridimensional y Cirugía Virtual para procedimientos Transobturatrizes. Actas Urológicas Españolas, v. 31, n. 4; p. 361-65, 2007. PETROS, P. E.; ULMSTEN, U. Na integral theory and its method for the diagnosis and management of female urinary incontinence. Scand. J. Urol. Nephorol, v. 153, p. 1-93,1993. PISANECHIE. Política Nacional de Saúde da Pessoa com Deficiência - Portaria MS/GM n. 1060 de 05/06/2002.Disponível em:<www.senado.gov.br/...

2009/ap20090707_erica_mini...>.

POLDEN, M.; MANTLE, J. Fisioterapia em Ginecologia e Obstetrícia. Editora Santos, 2002.

REIS, A.; SANTO, S.; DIAS, T. Estudo comparativo da capacidade de contração do assoalho pélvico em atletas de voleibol e basquetebol. 2008. 27f. In: RETT, M.T. et al. Qualidade de vida em mulheres apóstratamento da incontinência urinária de esforço com fisioterapia. Rev. Brasileira de Ginecologia e Obstetrícia, São Paulo, v.29, n. 3, p. 134-40, mar. 2007. RIOS, J. L.; SILVA, B.A. Fisiopatologia da incontinência urinária de esforço: artigo de revisão. Revista Digital. Bueno Aires, v. 14, n. 140, jan. 2010. 
SILVA, E.; SARRETA, F. O.; BERTANI, I. F. As políticas públicas de saúde no Brasil: o Sistema Único de Saúde (SUS) e a rede de saúde em Franca. Serviço Social \& Realidade, Franca, v. 16, n. 1, p. 81-103, 2007.

SIMÃO, R. Avaliação da medida simples da potência muscular máxima pelo fittrodyne.

Revista Brasileira de Atividade Física e Saúde, Londrina - PR, v. 6, n. 3, 2001.

TAVARES, M.B.A. C.; SOUSA, R.B.; SILVA, T.O.; MOREIRA, L.A.; SILVA, L.T.T.L.;

TAVARES, C.A.B.A.; VIEIRA, S.C. Prevalence of prognostic factors for câncer of the uterine cervix afther radical hysterectomy. Medical Journal, São Paulo, v. 127, n. 03, 2009. VIACAVA, F. Uma metodologia de avaliação do desempenho do sistema de saúde brasileiro. Ciência e Saúde Coletiva, v. 9, n. 3, p. 711-724, 2004.

VOLKMER, Cilene. Incontinência urinária feminina: revisão sistemática de estudos qualitativos. Ciência e Saúde Coletiva [online], v.17, n.10, p. 2703-2715, 2012. 